\title{
The indeterminist objectivity of quantum mechanics versus the determinist subjectivity of classical physics
}

\author{
Vasil Penchev, vasildinev@gmail.com \\ Bulgarian Academy of Sciences: Institute of Philosophy and Sociology: \\ Dept. of Logic and Philosophy of Science
}

\begin{abstract}
Indeterminism of quantum mechanics is considered as an immediate corollary from the theorems about absence of hidden variables in it, and first of all, the KochenSpecker theorem. The base postulate of quantum mechanics formulated by Niels Bohr that it studies the system of an investigated microscopic quantum entity and the macroscopic apparatus described by the smooth equations of classical mechanics by the readings of the latter implies as a necessary condition of quantum mechanics the absence of hidden variables, and thus, quantum indeterminism. Consequently, the objectivity of quantum mechanics and even its possibility and ability to study its objects as they are by themselves imply quantum indeterminism. The so-called free-will theorems in quantum mechanics elucidate that the "valuable commodity" of free will is not a privilege of the experimenters and human beings, but it is shared by anything in the physical universe once the experimenter is granted to possess free will. The analogical idea, that e.g. an electron might possess free will to "decide" what to do, scandalized Einstein forced him to exclaim (in a letter to Max Born in 2016) that he would be a shoemaker or croupier rather than a physicist if this was true. Anyway, many experiments confirmed the absence of hidden variables and thus quantum indeterminism in virtue of the objectivity and completeness of quantum mechanics. Once quantum mechanics is complete and thus an objective science, one can ask what this would mean in relation to classical physics and its objectivity. In fact, it divides disjunctively what possesses free will from what does not. Properly, all physical objects belong to the latter area according to it, and their "behavior" is necessary and deterministic. All possible decisions, on the contrary, are concentrated in the experimenters (or human beings at all), i.e. in the former domain not intersecting the latter. One may say that the cost of the determinism and unambiguous laws of classical physics, is the indeterminism and free will of the experimenters and researchers (human beings) therefore necessarily being out of the scope and objectivity of classical physics. This is meant as the "deterministic subjectivity of classical physics" opposed to the "indeterminist objectivity of quantum mechanics".
\end{abstract}

Keywords: choice, determinism and indeterminism free will, free will theorems, Kochen-Specker theorem

Prehistory, background and context:

Quantum mechanics studies (according to the fundamental postulate of Niels Bohr) the system of two kinds of entities, which seem to be contradictory at first glance: the discrete (quantum) and microscopic elementary particles, on the one hand, and the continuous (even smooth in the rigorous mathematical meaning) macroscopic apparatus, on the other hand, as a whole. The direct formal and logical contradiction, however, is avoided thanks to their "complementarity", another fundamental concept in quantum mechanics (and even not only), also suggested by Niels Bohr. Two complementary quantities cannot be measured simultaneously, particularly, a discrete (quantum) quantity and its counterpart being continuous. However, this means, furthermore, that any quantum quantity possesses a 
continuous complimentary counterpart sharing the same essence appearing alternatively being either quantum or continuous.

The avoided direct logical contradiction would imply the cancelation of quantum mechanics as a science for any conclusion would be "true" (and not less "false") in it. Quantum mechanics would not be able to state anything certainly. The concept of complementarity conserves it as a science, but at a certain cost: properly, that of quantum indeterminism:

Any result of quantum measurement is fundamentally random. This is a necessary condition for two contradicting properties such as discreteness and continuity to be unified by means of Bohr's complementarity. What is assignable unambiguously to any elementary particle is not a certain result of measurement (for it is fundamentally random), but the probability density distribution of all possible measurements of the particle at issue (or respectively, the characteristic function of that probability distribution which is an element of the separable complex Hilbert space of quantum mechanics, i.e. a wave function).

This a direct corollary from the theorems of the absence of hidden variables in quantum mechanics (such as, and first of all, the Kochen-Specker theorem). If those hidden variables could exist, discreteness and continuity would be commensurable therefore sharing them. As far as discreteness and continuity are contradictory to each other, they are furthermore fundamentally incommensurable to each other, and any hidden variable is impossible to exist in virtue of modus tollens. That is the essence of Kochen and Specker's proof. It hints the link of the ancient Pythagorean School's discovery of geometrical incommensurability and the theorem in question: properly, incommensurability (particularly, that of discreteness and continuity) implies trivially the absence of any shared standard (particularly, the suggestable hidden variables in quantum mechanics).

Further, the absence of hidden variables implies quantum indeterminism. Indeed, any repeatable (thus, not fundamentally random) result of quantum measurement can be considered as a "hidden variable" in the final analysis, and consequently, impossible.

Once the Kochen - Specker theorem and quantum indeterminism are granted as proved, the gates to the so-called free-will theorems are open already. Reflecting philosophically, the experiment's free will on the conditions of a few premises extracted from quantum mechanics and special relativity implies any elementary particle's "free will" (apropos, vice versa as well). One may speak of a fundamental and "subjectless" choice shared by the real subjects such as experimenters and the quasi-subjects such as elementary particles. That fundamental choice underlies the physical universe in virtue of quantum indeterminism and in turn, it suggests a special quantity of the number of elementary standard choices ("bits") such as the quantity of information.

That background and context is designated in the paper by the expression "indeterminist objectivity of quantum mechanics". The scope and intention of the paper is much modest and humble. If one means that indeterminist objectivity of quantum mechanics as a great achievement of physical science and even science at all as granted, how should one interpret classical mechanics and physics reversely on the same background?

\section{The thesis of the present paper is:}

The determinism of classical physics is more subjective and more anthropomorphic than the indeterminism of quantum mechanics for the former presupposes certain deterministic goal implicitly following the model of human freewill behavior. Quantum mechanics introduces the choice in the fundament of physical world involving a generalized case of choice, which can be called "subjectless": 
There are certain choices, which originate from the transition of the future into the past. Thus that kind of choice is shared of all existing and does not need any subject: It can be considered as a low of nature. There are a few theorems in quantum mechanics directly relevant to the topic: two of them are called "free will theorems" by their authors (Conway and Kochen 2006; 2009).

Any quantum system either a human or an electron or whatever else has always a choice: Its behavior is not predetermined by its past. This is a physical law. It implies that a form of information, the quantum information underlies all existing for the unit of the quantity of information is an elementary choice: either a bit or a quantum bit (qubit).

\section{A short comment of the thesis:}

A mathematically absolutely rigorous way for the "free will theorems" to be proved is demonstrated by the two scientists in their paper. Their conclusions can be grounded philosophically on the base of quantum holism, i.e. by means of the quantum and holistic unity of the quantum entity and experimenter. Choice is what really unifies all and can be interpreted as a kind of the solidarity of anything as being in time:

If the experimenter possesses "free will", the holistic unity implies the same for the electron. However, one can found the unity of the electron and human being in another way:

Both are forced to be "solidary" in front of the time, of the future and to share choice. Even more, they should be a coherent whole in their common future and only the choice is what can isolate them from each other in the present.

Classical physics remaining still anthropomorphic describes nature deterministically and similarly to human behavior. It conserves the choice ostensibly as a resource only for the human beings:

This is self-deception, which consists in the fundamental misunderstanding even of what a human being is and of how the human being differs from the rest in the universe.

The anthropomorphism of classical physics originates from the model of human behavior striving to certainty, determinability, and maximal control. Ostensibly only so, the human goals would be able to be achieved:

\section{Arguments for the thesis:}

1. Indeed quantum mechanics involves a generalized case of choice, which can be called "subjectless":

One might say that the apparatus is what chooses and thus quantum mechanics is fundamentally subjective unlike classical physics. Even more, quantum mechanics ostensibly needs a subject without whom reality might not exist. In fact, all those wrong conclusions originate from the misunderstanding of the subjectless choice involved by quantum mechanics.

2. The subjectless choice is what transforms future into past without the meditation of any subject of the choice. Its base is not any choosing whether a human being or an alleged "God", but mathematical laws or axioms such as the axiom of choice:

3. There is a fundamental kind of choice, which originates from the transition of the future into the past. This is the same choice introduced by quantum mechanics as the relation between the following two states:

3.1. The coherent state unorderable in principle for the theorems about the absence of hidden variables in quantum mechanics (Neumann 1932), (Kochen and Specker 1968);

3.2. The results of the measurement constituting always a well-ordered statistical ensemble.

4. The kind of subjectless choice is omnipresent just for no choosing to choose: 
Then one can say that all choose or the whole of all is what chooses:

5. That kind of choice is shared of all existing and does not need any subject:

The subjecteless choice of quantum mechanics can be deduced from the subjectless choice accompanying the course of time. Consequently, the time is what can relate to each other and even unify the subjectless choice in quantum mechanics and the freewill choice of a human being: Indeed any item in the universe should be adequate to the course of time in order to be able to exist.

6. Choice can be also considered as a universal law of nature as anything cannot help but choose for anything is in the present defined as the "medium of choice":

7. Choice can be considered even as the most universal law of nature. That law originates from time: So, choice should be a universal law not only of nature but not less, of consciousness, too. A human being unlike an electron possesses consciousness, but both share choice for both are relate to time. So, choice is the universal law of nature and consciousness.

8. Quantum mechanics was alleged to be dependent on an experimenter or even on an observer ostensibly deciding for the measured result what to be. This is not true, though. In fact, the determinism of classical physical deprives anything in the universe except the human being of the freedom of choice: a form of human chauvinism, subjectivism and anthropocentrism. This is wrong, too.

9 The human free will is limited by "categorical imperative" guaranteeing the free choice of anything in the universe. Consequently, the human free will is both only a form of the omnipresent choice and restricted by the same omnipresence of choice.

\section{The main conclusion:}

Quantum mechanics, on the one hand, and classical mechanics and physics (including even special and general relativity), on the other hand, realize two opposite strategies to the consistency and mutual possibility of freedom and necessity: a general philosophical problem embodied in the scientific and methodological problem of indeterminism and determinism accordingly:

Quantum mechanics for quantum indeterminism considers the formalized property of "free will" as shared by both "subjects" and "objects", both human beings and physical world.

On the contrary, classical physics distributes the same property disjunctively between the free subject and the rigorously deterministic object.

In fact, both solutions can be considered as decisions conditioned by the discreteness of quantum mechanics versus the continuity of classical physics. However, that of quantum mechanics cannot be accepted as more generalized for its condition is just the equivalence of discreteness and continuity implying in turn the equivalence of the two strategies how determinism and indeterminism to be consistent to each other. 


\section{References:}

Conway, J., Kochen, S. (2006) "The Free Will Theorem," Foundations of Physics 36 (10): 1441-1473.

Conway, J., Kochen, S. (2009) "The Strong Free Will Theorem," Notices of the AMS 56 (2): 226-232.

Kochen, S., Specker, E. (1968) "The problem of hidden variables in quantum mechanics," Journal of Mathematics and Mechanics 17 (1): 59-87.

Neumann, J. (1932) Mathematische Grundlagen der Quantenmechanik, Berlin, Springer. 